\title{
Controlling High PAPR in Vehicular OFDM-MIMO using Downlink Optimization Model under DCT Transform
}

\author{
Ahmed Ali ${ }^{1}$ \\ Department of Computer Science \\ College of Computer Engineering and Sciences, Prince \\ Sattam Bin Abdulaziz University \\ Al-kharj 11942, Saudi Arabia \\ Higher Future Institute for Specialized Technological \\ Studies, Cairo, Egypt
}

\author{
Esraa Eldesouky ${ }^{2}$ \\ Department of Computer Science \\ College of Computer Engineering and Sciences, Prince \\ Sattam Bin Abdulaziz University, Al-kharj 11942 \\ Saudi Arabia \\ Department of Computer Science, Faculty of Computers and \\ Informatics, Suez Canal University, Ismailia, Egypt
}

\begin{abstract}
The persisting challenges of the radio channel in vehicular networks entail the use of multi-antennas which is known as Multiple-Input Multiple-Output (MIMO). In order to obtain an efficient multi-user MIMO system, the power of the radio frequency (RF) components should be optimized. Necessarily practical solutions are essential to lower the vehicular nodes' complexity and support a robust Orthogonal Frequency Division Multiplexing (OFDM) discipline with a simplified equalization at the receiver. In this paper, the pre-coding ZadoffChu Sequence (ZCS) is employed along with the Discrete Cosine Transform (DCT) to control and optimize the high peak power. It intends the transmission over multi-user MIMO downlink vehicular channels. At last, the convex optimization is utilized to guarantee the peak-to-average power ratio (PAPR) minimization. Simulation results have shown that the proposed model can lessen the high PAPR compared to the least-square pre-coding. At the same time, it proved its effectiveness and accuracy as it enhances the transmission quality over multi-user MIMO-OFDM downlink vehicular channel.
\end{abstract}

Keywords-Zadoff-Chu Sequence; Discrete Cosine Transform (DCT); Peak-to-Average Power Ratio (PAPR); Vehicular Networks

\section{INTRODUCTION}

In vehicular networks, the Dedicated Short Range Communication (DSRC) protocol supports the vehicle-toroadside and vehicle-to-vehicle communications which enables numerous Intelligent Transportation Systems (ITS) applications $[1,2]$. However, the highly vehicular mobility affects the vehicular channel due to the frequency-selective multi-path propagation [3-7]. Thus, the variety of the available vehicular applications requires the use of efficient technologies such as OFDM-MIMO. This technology can boost the spectral efficiency by deploying several antennas at both the transmitter and the receiver [8].

The MIMO wireless communication technology allows the synchronization of large number of antennas at both sides while serving various vehicular users concurrently [9]. Among the privileges of employing MIMO are the efficient utilization of the RF, enhancement of the operational power consumption, and achievement of low complexity multi-user schemes. Multi- user MIMO communications is required in the vehicular networks due to the presence of huge node populations [9-13].

The OFDM vehicular channels usually suffer from the frequency selective fading, scattering, and high PAPR. As a result, the use of the linear power amplifiers is necessary to avoid out-of-band radiation and signal distortion [14, 15]. The emergence of curtailment of the high PAPR in the multi-user OFDM-MIMO can achieve better signal qualities and guarantee adequate data transmission.

Few research works have focus on the vehicular networks and their challenges [16-19]. Accordingly, the gap between MIMO and vehicular networks should be narrowed by offering an efficient channel modeling and estimation along with timely signal processing. In this paper, a novel vehicular model is proposed to control and optimize the PAPR resulted from applying the OFDM system on the transmitted signal. This model implements the DCT along with the ZCS pre-coding to enhance the signal quality in a vehicular downlink channel. The use of the ZCS combined with the DCT-OFDM can lower the complexity and cost of the high-power amplifier in a multiuser MIMO vehicular system.

This paper is organized as follows. Section 2 summarizes the literature review related to MIMO and vehicular networks. In Section 3, MIMO vehicular network is explained including the OFDM components and the ZCS pre-coding. However, Section 4 introduces the proposed IDCT MIMO-OFDM model. The channel downlink optimization is verified in Section 5. Section 6 introduces the experimental results and analysis for the proposed model. Finally, the paper is concluded in Section 7.

\section{RELATED WORKS}

Under the single-antenna wireless communication systems, several PAPR reduction solutions have been proposed [20-24]. Nevertheless, the PAPR reduction problem concerning the multi-user MIMO systems are described based on TomlinsonHarashima pre-coding, lattice pre-coding and joint pre-coding [25-27]. 
Christoph and Erik [27] proposed a PAPR reduction process by exploiting the excess of the transmitted antennas at the base station. The OFDM downlink transmission scheme is investigated for large-scale multi-user MIMO wireless systems to avoid expensive and inefficient power RF components at the base station. Yet, this work enlarges the processing complexity which makes it incompatible for the vehicular IEEE 802.11p channels [28]. The other two schemes [25, 26] based on Tomlinson-Harashima pre-coding require specialized signal processing algorithms at both terminals of the wireless link. This drawback prevents their implementation in wireless MIMO-OFDM systems, such as IEEE802.11n and 3GPP LTE. Similarly, they are inappropriate to vehicular networks as they increase the signal delay and latency.

An efficient detection ordering scheme in which the MIMO transmission is based on Zadoff-Chu Transform (ZCT) OFDM along with the root-raised cosine pulse shaping is presented in [29]. In [30], a joint pre-coding scheme is proposed for indoor downlink multi-user MIMO systems in which the terminals are able to receive spatially multiplexed symbol streams. Another spatial multiplexing multi-user MIMO downlink is proposed by Melvin et. al [31]. At this point, each user can receive multiple data sub-channels simultaneously using the same time and spectral resources.

Multi-user OFDM-MIMO communications are necessary to satisfy the requirements of any wireless communication system (i.e., vehicular networks) such as throughput and Quality-of-Service (QoS) demands. Since, vehicular networks and MIMO systems have been studied in several researches independently. Integrating such technologies can result in various benefits including significant improvement in the range and reliability of the vehicular communication via beamforming. Furthermore, the network throughput can be enhanced when using MIMO technology due to the efficient management of the multiuser interference.

IEEE 802.11p standard does not come with any specification for a vehicular MIMO version. Accordingly, researchers pay more attention to capture the benefits of using multiple antennas in vehicular environment [32]. Thus, the vast vehicular applications require a joint optimization for the medium access control (MAC), network and physical layers.

Sending dependent signals through multi-paths is considered the main clue for MIMO technology. Though, several replicas of the data symbol can be obtained at the receiver leading to a reliable diversity gain. Therefore, multiple parallel subchannels can be obtained (i.e., spatial dimension). Consequently, the link capacity can be enhanced by transmitting independent replicas of data symbols in parallel manner through orthogonal subchannels.

\section{MIMO VEHICULAR NETWORKS}

With the increasing number of vehicular activities and drivers' demands, more adequate propagating channels should be guaranteed. The key idea is to enhance transportation safety, efficiency and stillness through the well-use of the short connectivity time among vehicular nodes. The availability of efficient vehicular channels can ensure the arrival of the warning alerts and safety messages between vehicles and roadside infrastructures. Consequently, traffic congestion, fatalities or any accidental injuries can be avoided.

Sending dependent signals through multi-paths is considered the main clue for MIMO technology. Though, several replicas of the data symbol can be obtained at the receiver leading to a reliable diversity gain. Therefore, multiple parallel sub-channels can be obtained (i.e., spatial dimension). Consequently, the link capacity can be enhanced by transmitting independent replicas of data symbols in parallel manner through orthogonal sub-channels.

\section{A. Peak-to-Average Power Ratio in OFDM Model}

The OFDM signal can suffer from high PAPR which should be reduced in order to improve the transmission quality and lower the system complexity. The resulted high PAPR can lead to nonlinear impacts on the wireless OFDM signal as well as distortion in the electrical equipment. Thus, an efficient OFDM channel with controlled PAPR should be suggested to cope with the fast-changeable vehicular channel.

According to [33], PAPR is the relationship between the maximum power of a transmitted OFDM sample and its average power as shown in equation (1).

$$
P A P R=10 \log _{10} \frac{\text { Power }_{\text {pak }}}{\text { Power }_{\text {aerenge }}}(\mathrm{dB})
$$

Where Power $_{\text {peak }}$ and Power $_{\text {verage }}$ are the peak and average power of a transmitted OFDM symbol, respectively. They can be estimated using equations (2) and (3).

$$
\begin{aligned}
& \text { Power }_{\text {peak }}=\max _{0 \leq t \leq L T_{s}}|x(t)|^{2}, \\
& \text { Power }_{\text {average }}=\frac{1}{L T_{s}} \int_{0}^{T_{s}}|x(t)|^{2},
\end{aligned}
$$

Where $L$ is the number of the modulated subcarriers, $T_{s}$ is the sampling period, and $x(t)$ is the OFDM input signal over time $t$.

Hence, a high PAPR appears when the subcarriers of a given OFDM symbol are out of phase with each other (i.e., the use of a large number of subcarriers). Actually, it is one of the main challenges in any multi-carrier system using the OFDM MIMO.

\section{B. Zadoff-Chu Sequence Pre-coding}

It is necessary to reduce and control the PAPR of the transmit signal in the proposed vehicular OFDM-MIMO system through a pre-coding techniques. In the proposed model, the ZCS pre-coding technique is deployed to reshape the IDCT matrix row and reform the constellation symbols before the channel propagation step [34]. The main advantages of ZCS is its ability to provide constant amplitude for the output signal with optimum correlation properties as well as reduce the cost and complexity of output signal $[35,36]$. Thus, the peak power of the transmitted signal can be under control. A ZCS $z$ of length $L_{s}$ can be mathematically defined as: 


$$
z(n)= \begin{cases}e^{\frac{j 2 r \pi}{L_{s}}\left(\frac{k^{2}}{2}+q k\right)} & \text { for } L_{s} \in \text { even }, \\ e^{\frac{j 2 r \pi}{L_{s}}\left(\frac{k(k+1)}{2}+q k\right)} & \text { for } L_{s} \in \text { odd }\end{cases}
$$

\section{PRoposed InVERSE Discrete Cosine TRANSFORM OFDM-MIMO MODEL}

MIMO technique was firstly introduced by Goldsmith et al. in [37], which addresses the multi-antennas IEEE 802.11 wireless networks. Mainly, it supports the multi-user case using similar capacity. In other words, a MIMO system with $M$ transmit antennas and $N$ receive antennas can have a linear capacity of $\min (M, N)$. In general, the multi-user MIMO downlink technology is applicable to several wireless networks with multi-antenna infrastructures such as base stations or access points. In the proposed model, a DCT transformation mechanism is considered based on a multi-user OFDM-MIMO using the ZCS pre-coding over vehicular downlink channels in order to decrease the resulted PAPR and enhance the network reliability.

The proposed model considers a roadside unit infrastructure with a large number of transmit antennas $M$ that serve multi-user receivers $N$ where $M \geq N$. The receiver antennas are treated as independent terminals (i.e., vehicular nodes) whereas each terminal has its own antenna. The vehicular MIMO channel has the form of matrix $H$ with $N \times M$ dimension. $[H]_{i, j}$ is the channel gain (i.e., transfer function) from the $j^{\text {th }}$ transmitter antenna to the $i^{\text {th }}$ receiver antenna. The transmitted signal $x$ is defined to be a complex vector that collects all the transmitted MIMO-OFDM symbols such that $x=\left[x_{0}, x_{1}, \ldots, x_{M-1}\right]^{T}$. Though, $x_{j}$ is the transmitted symbol from the $j^{\text {th }}$ transmit antenna; where $j=0,1, \ldots, M-1$ . The input-output relationship of the channel matrix $H$ is computed as in equation 9.

$y=H x+w$,

Where $w=\left[w_{1}, w_{2}, \ldots, w_{N}\right]^{T}$ is an one-dimensional vector of Gaussian additive noise; and $y=\left[y_{1}, y_{2}, \ldots, y_{N}\right]^{T}$ is the vector of the received data. The elements of the noise vector follow complex zero mean Gaussian distribution with variance $\sigma^{2}$. Since, this model deals with multi-user vehicular nodes, the $N$ receivers are assigned to different users. Thus, each vehicular user has access to only one element of vector $y$.

This model considers a case that the roadside unit attempts to transmit replicas of the digital signal to multiple vehicular nodes (i.e., spatial diversity mode). For example, broadcasting a safety message, traffic status, or even an advertisement. According to Fig. 2, a roadside unit (RS) commences a broadcast action whereas a transmitted digital signal $x$ is ready for propagation. Firstly, the replicas of the transmitted signal are created using the diversity processor block. Then, they are converted from serial to parallel form using the serial-toparallel (S/P) converter in order to be sent through the roadside antennas. At this moment, the resulted sequence is defined as $A=\left[A_{0}, A_{1}, \ldots, A_{L-1}\right]$ based on the one-to-one mapping and the number of the received antennas $N$.

Fig. 1. Transmitted Signal over the Vehicular Channel. 
The DCT/IDCT transform is employed in this model [22, 36, 38]. Similar to other transformation methods, the DCT relies on a sum of cosine function that is fluctuating at different frequencies to define a finite sequence of data points. The cosine functions can particularly express the choice of the boundary conditions. Moreover, DCT transform allows a digital signal to be propagated efficiently in the frequency domain. Each OFDM-MIMO signal (i.e., $A$ ) is passed through the inverse DCT (IDCT) block to generate the complex baseband OFDM signal with subcarriers according to Equations (10) and (11).

$$
a(k)=\left\{\begin{array}{lr}
s(k) \sum_{i=0}^{L-1} A(n) \cos \left[\frac{\pi(2 l+1) k}{2 L}\right] & \text { for } 0 \leq k \leq L, \\
0 & \text { Otherwise }
\end{array}\right.
$$

$$
s(k)= \begin{cases}\frac{1}{\sqrt{L}} \text { for } & \text { for } k=0, \\ \frac{2}{\sqrt{L}} & \text { for } k \neq 0\end{cases}
$$

Where $L$ is the length of the OFDM symbols to get the time domain samples $a=\left[a_{1}, a_{2}, \ldots, a_{N}\right]^{T}$.

After The IDCT transform, the Cyclic Prefix (CP) inserted to the samples. Then, the baseband is converted to passband signal using the Up-Frequency Conversion (UFC). Afterwards, the signal is pre-coded with the ZCS transform and passed over the IEEE 802.11p vehicular channel [28].

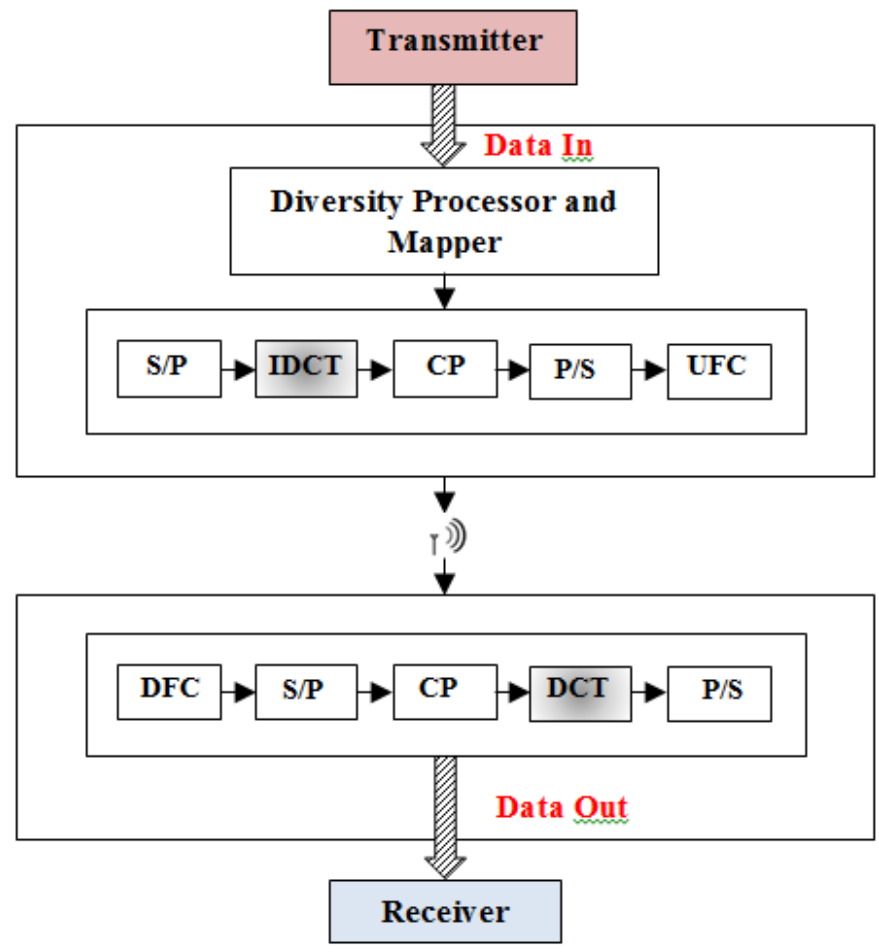

Fig. 2. The Proposed OFDM-MIMO Downlink Model.
At the receiver antennas, the passband signal is reconverted to baseband signal using the Down-Frequency Conversion (DFC).The DCT transform is applied to the received OFDM symbols. Finally, the signal is received through the vehicular users' antennas. The basic main steps of the proposed model can be summarized as follows:

Step1: An RSU with antennas transmits a digital signal to multi-vehicular users whereas each with single independent antenna.

Step2: The sent signal is converted to parallel subcarriers, transformed using the IDCT block, and cyclic prefix is added to avoid the intersymbol interference

Step3: The transmitted signal is then transformed to passband using the UFC.

Step4: ZCS pre-coding is applied to the transmitted signal at the roadside unit in order to control the PAPR.

Step5: The signal is propagated over the vehicular channel, and exposed to some noise.

Step6: At the receiver side, the baseband signal is obtained by using the DFC followed by the removal of the cyclic prefix and the signal is re-converted to serial form as to be received by the users' antennas.

This combinational model aims to employ the ZCS precoding and the DCT/IDCT transform to reduce the resulted PAPR in MIMO-OFDM downlink model.

\section{CHANNEL DOWNLINK OPTIMIZATION}

As aforementioned, the proposed model aims to control the dynamic range while transmitting the input signal $A$. This signal is exposed to a channel impulse response $H$, as illustrated in equation (9). Given a constraint that the number of the transmit antenna is larger than or equal to the receive antenna. The ZCS pre-coding is employed to the input signal $A$ in an effort to reduce the PAPR and get $A^{\prime}$. Hence, to avoid the multi-user interference, $A^{\prime}$ must satisfy the pre-coding constraint $A^{\prime}=H A$, which ensures $y=A+w$. This means that the pre-coding must be applied at the transmitter side (i.e., roadside unit).

In general, the primary objective is to find an appropriate $A^{\prime}$ which ensures a small dynamic range and optimized PAPR. This can be obtained by employing the following objective function:

$$
\left(P-D y n-A^{\prime}\right) \quad \min \quad \alpha-P A P R_{A}
$$

Subject to $A^{\prime}=H A$

And $\quad P A P R_{A} \leq\left|A_{m}^{\prime}\right| \leq \alpha \quad m=1, \ldots, B$

Where $\left(P-D y n-A^{\prime}\right)$ is the optimization objective that aims to control the PAPR values of $A^{\prime}, P A P R_{A}$ is the actual PAPR value of the OFDM symbol, $B$ is the number of the transmitted OFDM symbols and $\alpha$ is the desired PAPR 
threshold. This means that $\left(P-D y n-A^{\prime}\right)$ exhibits optimized lower PAPR than the transmit vector $A^{\prime}$ resulted from the ZCS pre-coding block. However, satisfying the inequality $P A P R_{A} \leq\left|A_{m}^{\prime}\right| \leq \alpha$ seems to be difficult. Then, $\left(P-D y n-A^{\prime}\right)$ needs to be relaxed by only considering the upper bound constraint $\left|A_{m}^{\prime}\right| \leq \alpha$.

Thus, the convex optimization problem can be reformulated as follows:

$$
(P-D y n-R) \underline{\text { minimize }} \max \left\|A_{m}^{\prime}\right\|_{B} \text { for } m=1, \ldots, B,
$$

Subject to

$A^{\prime}=H A$

At this moment, the $(P-D y n-R)$ is expected to minimize the magnitude of the largest entry in $A^{\prime}$ which consequently ensures lower PAPR values. This single optimization problem is solved using the convex optimization which mainly studies the problem of minimizing the convex function over convex sets. Thus, the convex optimization function can be re-defined as follows:

$$
\left(P-D y n-a^{\prime}\right) \min _{a_{1}, a_{2}^{\prime}, \ldots, a_{B}^{\prime}} \max \left\{\left\|a_{1}^{\prime}\right\|,\left\|a_{2}^{\prime}\right\|, \ldots,\left\|a_{B}^{\prime}\right\|\right\}
$$

Subject to

$$
a_{b}^{\prime}=H_{b} a_{b} \quad \text { for } \quad b=1,2, \ldots, B
$$

Where, the vector $a_{b}^{\prime}$ corresponds to the pre-coded OFDM symbols that are transmitted from each antenna to be minimized.

Thereafter, the result PAPR values of the optimized symbols $(P-D y n-R)$ and the PAPR of the ZCS pre-coding should satisfy the following equality:

$(P-D y n-R)=\frac{N\left\|O P_{A}\right\|_{B}^{2}}{\left\|O P_{A}\right\|_{2}^{2}} \leq \frac{N\left\|A^{\prime}\right\|_{B}^{2}}{\left\|A^{\prime}\right\|_{2}^{2}}=P A P R_{Z C S}$

This implies that the PAPR of the convex optimization cannot exceed the PAPR values of the ZCS pre-coding technique for all the transmitted OFDM symbols.

According to proposition 1 in [27], which states that:

"If $H$ has the full column rank and $1<M<N$-given that $M, N$ are integers- then, the solution $O P_{A}$ of the $(P-D y n-R)$ can have $N-M+1$ entries with a magnitude equal to $\left\|O P_{A^{\prime}}\right\|_{B}$."

The upper bound of the $P A P R_{(P-D y n-R)}$ is given by:
$(P-D y n-R)=\frac{N\left\|O P_{A}\right\|_{B}^{2}}{\left\|O P_{A}\right\|_{2}^{2}} \leq \frac{N}{N-M+1}$

Such that:

$$
\begin{aligned}
& \left\|O P_{A}\right\|_{2}^{2}=\sum_{\mu}\left\|O P_{A}\right\|_{B}^{2}+\sum_{i \in \mu}\left|\left[O P_{A^{\prime}}\right]_{i}\right|^{2} \\
& \geq \sum_{\mu}\left\|O P_{A^{\prime}}\right\|_{B}^{2}=(N-M+1)\left\|O P_{A^{\prime}}\right\|_{B}^{2}
\end{aligned}
$$

Where $\mu$ is the indices for the $N-M+1$ entries of $a^{\prime}$ for which $\left|\left[O P_{A^{\prime}}\right]_{i}\right|=\left\|O P_{A^{\prime}}\right\|_{B}$. As a result, the $P A P R_{(P-D y n-R)}$ can approach to 1 for a predefined number of antennas $M$ and $N$. This means that the $P A P R_{(P-D y n-R)}$ can eliminate the multi-user interference while keeping constant envelope signal. As a result, these transmitting vectors can succeed to have low PAPR.

\section{SimULATION RESULTS AND ANALYSIS}

The effectiveness and accuracy of the proposed model is demonstrated via simulation using MATLAB. As previously mentioned, this model considers the PAPR reduction problem in OFDM-MIMO vehicular networks. It is a downlink scheme that mimics the transformation of a digital signal from a multiantennas roadside unit to independent multi-users. The DCT transformation is used instead of the Fast Fourier Transform (FFT) along with a ZCS pre-coding in order to reduce the PAPR and system complexity.

\section{A. Simulation Setup}

According to Studer and Larsson [27], the proposed model assumes a roadside unit with 100 antennas installed and 10 vehicular receivers. Nevertheless, the number of receivers is increased to reach 30 end-users. It is known that the IEEE $802.11 \mathrm{p}$ amendments are basically based on the OFDM scheme [28]. Thus, an OFDM system with 64 subcarriers is employed [39], a bandwidth of $10 \mathrm{MHz}$, and a transmission data rate of $27 \mathrm{Mbps}$ as specified in the IEEE 802.11p. The cyclic prefix is of the length 16 along with $480 \mathrm{~m}$ propagation distance whereas the excess delay is given by $6 \mu \mathrm{s}$.

In the proposed model, each user is assigned to a coded transmission with 216 information bits. These encoded bits are mapped using 16-QAM constellation as well as a convolutional code with coding rate of $1 / 2$ and constraint length of 7 [40].

The vehicular channel is considered as a frequencyselective multipath channel. Hence, the pre-coded signal resulted from the ZCS pre-coding block is transmitted in the form of a tap-delay line with 4 taps. At the end, the transmitted signal is detected at the receiver side (i.e., independent vehicular users) which performs a demodulation process and applies the DCT transform. The proposed model is applied on the transmitted OFDM-MIMO-symbols in order to reduce the PAPR ratio. Table I lists the parameters values used in the conducted simulations. 
TABLE. I. SimULATION PARAMETERS AND VALUES

\begin{tabular}{|l|l|}
\hline Items & Values \\
\hline Number of Subcarriers & 64 \\
\hline Data Subcarriers & 52 \\
\hline Cyclic Prefix & 16 \\
\hline Modulation & 16 -QAM \\
\hline Number of taps & 4 \\
\hline Transmit antennas & 100 \\
\hline Receive antennas & $10,20,30$ \\
\hline Transmission Data Rate & $27 \mathrm{Mbps}$ \\
\hline Excess Delay & $6 \mu \mathrm{s}$ \\
\hline Coding rate & $1 / 2$ \\
\hline Constraint Length & 7 \\
\hline
\end{tabular}

To evaluate the performance of the proposed model, the Complementary Cumulative Distribution Function (CCDF) is used. The CCDF metric computes the probability of the OFDM signal exceeding the predefined PAPR threshold within the OFDM symbols and it is given by:

$$
C C D F\left[P A P R_{i}\right]={ }_{P r o b}\left[P A P R_{i}>P A P R_{0}\right]
$$

Where, $P A P R_{0}$ is the PAPR threshold and $P A P R_{i}$ is the PAPR value of the $i^{\text {th }}$ OFDM symbol in the transmitted signal.

In addition, the average symbol-error rate is measured in order to estimate the performance bits error rate across the vehicular users. A symbol is considered to have an error if the received symbols are different from the transmitted symbols. As the result, the transmitted information bits are changed. Then, the average symbol-error rate $\left(A V G_{S E R}\right)$ is given below:

$A V G_{S E R}=\frac{\sum_{i=1}^{V_{N}} S E R_{i}}{V_{N}}$

Where, $S E R_{i}$ is the symbol-error rate for the receiver overall the vehicular receivers $V_{N}$.

\section{B. Results Analysis}

In this simulation, the proposed model is compared with the Least-Square pre-coding technique [41] and the classical OFDM system without using pre-coding techniques. The experiments are conducted in a downlink scheme whereas various receiver populations are used.

Fig. 3, 4 and 5 show the CCDF of the PAPR distribution where 16-QAM is applied to this model.

In Fig. 3, the PAPR values illustrate the use of various precoding techniques using 10 independent receiving antennas (i.e., vehicular users). The target PAPR of the proposed model is obviously decreased compared to the other two techniques due to applying of the ZCS pre-coding technique along with the DCT transform. The maximum reached PAPR value for the proposed model is $6.22 \mathrm{~dB}$. However, the conventional OFDM (i.e., without a PAPR reduction mechanism) and the LeastSquare pre-coding [42] are $10.35 \mathrm{~dB}$ and $12.51 \mathrm{~dB}$, respectively.

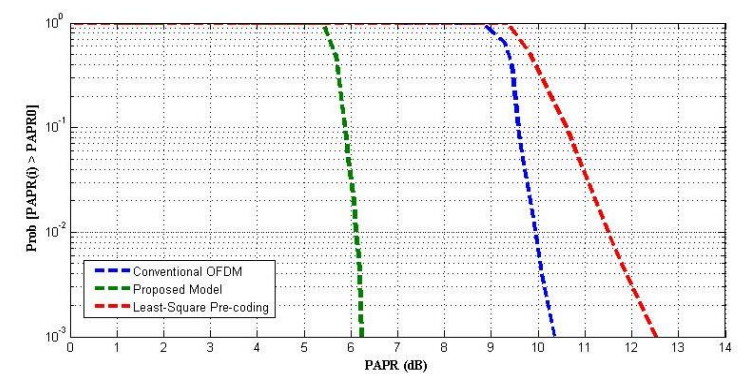

Fig. 3. PAPR Performance using DCT Transform in Vehicular Networks.

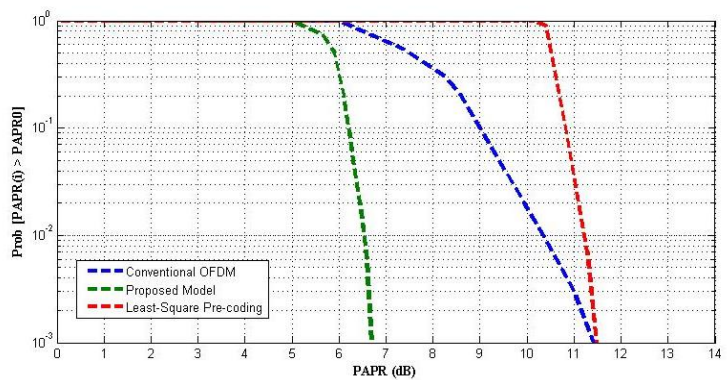

(a)

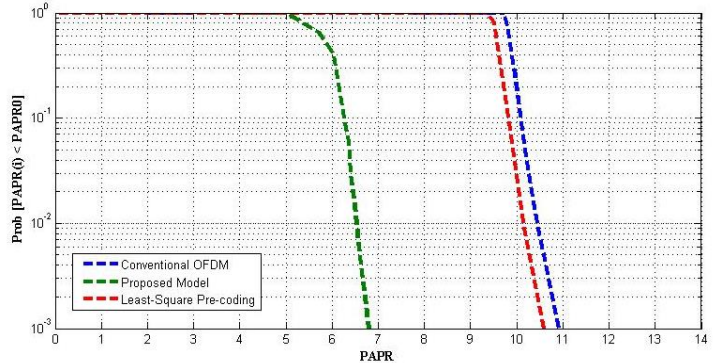

(b)

Fig. 4. PAPR CCDF using the ZCT Pre-Coding in a MIMO Downlink Vehicular Channel.

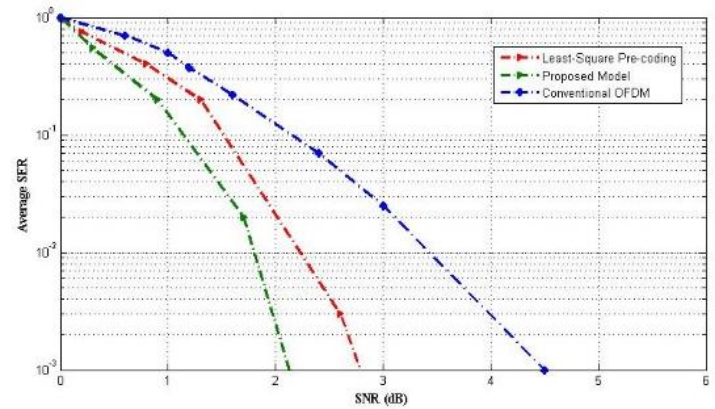

Fig. 5. Average SER using 16-QAM and 10 Receive Vehicular Antennas.

Nevertheless, the PAPR of the tested vehicular channel is effectively decreased when the number of receivers is increased to reach 20 and 30 vehicular users as respectively shown in Fig. 4(a) and (b). The Least-Square pre-coding is affected by the antennas population so that if the number of the network nodes increases, the associated PAPR increased either. The maximum PAPR when using the Least-Square Pre-coding are 11.48 and $10.59 \mathrm{~dB}$, respectively. Nevertheless, the PAPR for the proposed model is reduced by $5 \mathrm{~dB}$ when compared to the other two schemes. 
According to the simulation results, the proposed model (i.e., ZCS pre-coding plus DCT transform) succeeds to reduce the high PAPR resulted due to the use of OFDM- MIMO system in the vehicular networks. The received time-domain signal has a significant smaller PAPR than of the Least-Square (i.e., up to $6 \mathrm{~dB}$ ).

The Symbol-Error-Rate (SER) is the probability of receiving a symbol in error. It is usually calculated by comparing the ratio of symbols in error to the total number of bits. Hence, the SER performance is studied for the proposed pre-coding downlink model, Least-Square pre-coding and the conventional OFDM. In this paper, the SER has been estimated with respect to different Signal-to-Noise Ratio (SNR) to determine the information bits which are affected during transmission (i.e., symbols that are not correctly received).

Fig. 5 shows the SER performance of the three models. The horizontal axis indicates the SNR in $\mathrm{dB}$ and the vertical axis represents the average SER. It demonstrates that the increase in the SNR values lead to lower average SER for the three schemes using 16-QAM modulation and 10 receiver antennas. Moreover, it has been observed that the proposed model provides the best SER compared to the Least-Square precoding.

According to the simulation results, the proposed model that relies on ZCS pre-coding and DCT transform in an OFDM-MIMO vehicular channel succeeds to significantly reduce both the PAPR and the SNR for the transmitted symbol.

\section{VII.CONCLUSIONS}

Since the IEEE $802.11 \mathrm{p}$ standard is mainly designed for low complexity receivers in vehicular networks, a degraded performance can occur due to the high PAPR resulted from using the OFDM system. Hence, a low packet error rate is crucial for specifically the safety-related scenarios. The use of multiple antennas at both the transmitter and the receiver may improve the network throughput but may result in a multi-user interference problem. This can be resolved using a multi-user OFDM -MIMO system. In this paper, a joint multi-user OFDM-MIMO model based on the ZCS pre-coding combined with DCT/IDCT transform is proposed. This model aims to control and reduce the high PAPR over the vehicular channel in a downlink vehicle-to-roadside environment. According to the conducted simulations, the results show that the proposed model improves the vehicular channel performance by decreasing the PAPR. This model achieves observable lower PAPR compared to the Least-Square pre-coding and the conventional OFDM. Using 10, 20 and 30 receive independent antennas; the PAPR for the proposed model is $6.22 \mathrm{~dB}, 6.69$ $\mathrm{dB}$, and $6.79 \mathrm{~dB}$, respectively. Accordingly, this model improves the transmission quality over multi-user OFDMMIMO downlink vehicular channel.

Although, vehicular networks acquire numerous privileges of the presence and utilization of multiple antennas, challenges regarding PAPR in MIMO vehicular networks still exist. A future pathway is the proper analysis of the imperfect Channel State Information (CSI) effect using the ZCS pre-coding for vehicular communication. Efficient metaheuristic optimization algorithms can be implemented in order to powerfully minimize the PAPR values for the input signal. Finally, the nonstationary nature of vehicular topology requires analyzing the channel estimation technique recursively in order to track the impulse response of the MIMO channel.

\section{ACKNOWLEDGMENT}

We would like to acknowledge with much appreciation Prince Sattam Bin Abdulaziz University for its ongoing support to our research.

\section{REFERENCES}

[1] P. Mutalik, and V. C. Patil, "A survey on vehicular ad-hoc network [VANET's] protocols for improving safety in urban cities." International Conference On Smart Technologies For Smart Nation (SmartTechCon), Bangalore, pp. 840-845, August 2017.

[2] E. Eldesouky, W. Elashmawi, L. Renfa, and W. Dong, "A Hybrid Cooperative Model for Bandwidth Utilization in Vehicular Ad hoc Networks Based on Game Theory," International Journal of Control Automation, vol. 7, no. 12, pp. 177-188, 2014.

[3] B. Bloessl, F. Klingler, F. Missbrenner, and C. Sommer, "A systematic study on the impact of noise and OFDM interference on IEEE 802.11p.", IEEE Vehicular Networking Conference (VNC), Torino, pp. 287-290, Novemeber 2017.

[4] A. Ali, W. Dong, L. Renfa et al., "ISI and PAPR Immune IEEE $802.11 \mathrm{p}$ Channels Based on Single-Carrier Frequency Domain Equalizer," KSII Transactions on Internet Information Systems, vol. 10, no. 11, pp. 55135529, 2016.

[5] U.A. Khan, and S.S. Lee, " Multi-Layer Problems and Solutions in VANETs: A Review," Electronics, vol. 8, no. 2, pp. 1-19, 2019.

[6] L. Liang, H. Peng, G.Y. Li, and X. Shen, " Vehicular communications: A physical layer perspective,", IEEE Transactions on Vehicular Technology, vol. 66, no.12, pp. 10647-10659, 2017.

[7] L. Cheng, W. Viriyasitavat, M. Boban, and H.M. Tsai, "Comparison of radio frequency and visible light propagation channels for vehicular communications," IEEE Access, vol. 6, pp. 2634-2644, 2018.

[8] N. Adhikari, "Antenna selection and MIMO capacity estimation for vehicular communication systems" The University of North Dakota, Theses and Dissertations, 2014.

[9] S. T. Abraha, D. F. Castellana, X. Liang, A. Ng'oma, and A. Kobyakov, "Experimental Study of Distributed Massive MIMO (DM-MIMO) in Inbuilding Fiber-Wireless Networks.", Optical Fiber Communications Conference and Exposition (OFC), San Diego, pp. 1-3, March 2018.

[10] H. Q. Ngo, E. G. Larsson, and T. L. Marzetta, "Energy and spectral efficiency of very large multiuser MIMO systems," IEEE Transactions on Communications, vol. 61, no. 4, pp. 1436-1449, 2013.

[11] F. Rusek, D. Persson, B. K. Lau, E.G. Larsson, T.L. Marzetta, O. Edfors, and F. Tufvesson, "Scaling up MIMO: Opportunities and challenges with very large arrays," IEEE Signal Processing Magazine, vol. 30, no. 1, pp. 40-60, 2013.

[12] J. Zhang, Y. Huang, J. Wang et al., "Per-antenna constant envelope precoding and antenna subset selection: A geometric approach," IEEE Transactions on Signal Processing, vol. 64, no. 23, pp. 6089-6104, 2016.

[13] A. El-Keyi, T. ElBatt, F. Bai, and C. Saraydar, "MIMO VANETs: Research challenges and opportunities.", International Conference on Computing, Networking and Communications (ICNC), Maui, pp. 670676, January 2012.

[14] A. Chadha, N. Satam, and B. Ballal, "Orthogonal frequency division multiplexing and its applications," International Journal of Science and Research (IJSR), vol. 2, no. 1, 2013.

[15] S. Bachir, B. Koussa, C. Perrine, C. Duvanaud, and R. Vauzelle, "Peak power reduction for OFDM systems in vehicular wireless communications context.", Proceedings of the International Conference on ELECTRONICS, COMPUTERS and ARTIFICIAL INTELLIGENCE - ECAI-2013, Pitesti, pp. 1-9, June 2013.

[16] T. Abbas, J. Nuckelt, T. Kürner, T. Zemen, C.F. Mecklenbräuker, and F. Tufvesson, " Simulation and measurement-based vehicle-to-vehicle channel characterization: Accuracy and constraint analysis," IEEE 
Transactions on Antennas and Propagation, vol. 63, no. 7, pp. 3208-3218, 2015.

[17] A. Attia, A. A. ElMoslimany, A. El-Keyi, T. ElBatt, F. Bai, and C. Saraydar, "MIMO vehicular networks: Research challenges and opportunities," Journal of communications, vol. 7, no. 7, pp. 500-513, 2012.

[18] S. Fikar, W. Walzik, and A. L. Scholtz, "Vehicular multi/broadband MIMO antenna for terrestrial communication." IEEE Antennas and Propagation Society International Symposium, Toronto, pp. 1-4, July 2010.

[19] F. Hu, Vehicle-to-vehicle and Vehicle-to-infrastructure Communications: A Technical Approach: CRC Press, 2018.

[20] R. J. Baxley, and G. T. Zhou, "Comparing selected mapping and partial transmit sequence for PAR reduction," IEEE Transactions on Broadcasting, vol. 53, no. 4, pp. 797-803, 2007.

[21] N. I. Miridakis, and D. D. Vergados, "A survey on the successive interference cancellation performance for single-antenna and multipleantenna OFDM systems," IEEE Communications Surveys Tutorials, vol. 15, no. 1, pp. 312-335, 2013.

[22] S. Abouty, L. Renfa, Z. Fanzi, and F. Mangone, "A novel iterative clipping and filtering technique for PAPR reduction of OFDM signals: system using DCT/IDCT transform," International Journal of Future Generation Communication, vol. 6, no. 1, pp. 1-8, 2013.

[23] A. Gangwar, and M. Bhardwaj, "An overview: Peak to average power ratio in OFDM system \& its effect," International Journal of Communication Computer Technologies, vol. 1, no. 2, pp. 22-25, 2012.

[24] G. Wunder, R. F. Fischer, H. Boche et al., "The PAPR problem in OFDM transmission: New directions for a long-lasting problem," IEEE Signal Processing Magazine, vol. 30, no. 6, pp. 130-144, 2013.

[25] S. Umeda, S. Suyama, H. Suzuki, K. Fukawa, "PAPR reduction method for block diagonalization in multiuser MIMO-OFDM systems." IEEE 71st Vehicular Technology Conference, pp. 1-5, May 2010.

[26] C. Siegl, and R. F. Fischer, "Selected basis for PAR reduction in multiuser downlink scenarios using lattice-reduction-aided precoding," EURASIP Journal on advances in signal processing, vol. 2011, no. 1, pp. 17, 2011.

[27] C. Studer, and E. G. Larsson, "PAR-aware large-scale multi-user MIMOOFDM downlink," IEEE Journal on Selected Areas in Communications vol. 31, no. 2, pp. 303-313, 2013.

[28] D. Jiang, and L. Delgrossi, "IEEE 802.11 p: Towards an international standard for wireless access in vehicular environments.", VTC Spring 2008 - IEEE Vehicular Technology Conference, Singapore, pp. 20362040, May 2008.

[29] V. Sreevani, and K. Suma, "Reduction of PAPR and Efficient detection ordering scheme for MIMO Transmission Systems Using Power Control," International Journal of Engineering Research Applications, vol. 5, no. 12, pp. 31-35, 2015.
[30] Q. Zhao, Y. Fan, and B. Kang, "A joint precoding scheme for indoor downlink multi-user MIMO VLC systems," Optics Communications, vol. 403, pp. 341-346, 2017.

[31] M. C. Lim, M. Ghogho, and D. C. McLernon, "Spatial multiplexing in the multi-user MIMO downlink based on signal-to-leakage ratios.", IEEE Global Telecommunications Conference, Washintgon, pp. 3634-3638, November 2007.

[32] S. Moser, L. Behrendt, and F. Slomka, "MIMO-enabling PHY layer enhancement for vehicular ad-hoc networks," IEEE Wireless Communications and Networking Conference Workshops (WCNCW), New Orleans, pp. 142-147, March 2015.

[33] T. Jiang, and Y. Wu, "An overview: Peak-to-average power ratio reduction techniques for OFDM signals," IEEE Transactions on broadcasting, vol. 54, no. 2, pp. 257-268, 2008.

[34] I. Baig, and V. Jeoti, "On the PAPR reduction in OFDM systems: a novel ZCT precoding based SLM technique," Journal of Engineering Science Technology, vol. 6, no. 3, pp. 357-368, 2011.

[35] I. Baig, and V. Jeoti, "Zadoff-Chu matrix transform precoding-based orthogonal frequency division multiple access uplink systems: a peak-toaverage power ratio performance," Arabian Journal for Science Engineering, vol. 38, no. 3, pp. 613-620, 2013.

[36] A. Ali, W. Dong, and L. Renfa, "A Novel ZCS-Clipping Precoding Technique for PAPR Reduction using DCT-OFDM in Vehicular Channels," International Journal of Hybrid Information Technology, vol. 8, no. 5, pp. 11-22, 2015.

[37] A. Goldsmith, S. A. Jafar, N. Jindal, and S. Vishwanath, "Capacity limits of MIMO channels," IEEE Journal on selected areas in Communications, vol. 21, no. 5, pp. 684-702, 2003.

[38] F. Mangone, J. He, J. Tang, J. Xiao, M. Chen, F. Li, and L. Chen, "A PAPR reduction technique using Hadamard transform combined with clipping and filtering based on DCT/IDCT for IM/DD optical OFDM systems," Optical Fiber Technology, vol. 20, no. 4, pp. 384-390, 2014.

[39] C. F. Mecklenbrauker, A. F. Molisch, J. Karedal, F. Tufvesson, A. Paier, L. Bernadó, T. Zemen, O. Klemp, and N. Czink, "Vehicular channel characterization and its implications for wireless system design and performance," Proceedings of the IEEE, vol. 99, no. 7, pp. 1189-1212, 2011.

[40] I. Baig, and V. Jeoti, "PAPR reduction in OFDM systems: Zadoff-Chu matrix transform based pre/post-coding techniques.", 2nd International Conference on Computational Intelligence, Communication Systems and Networks, Liverpool, pp. 373-377, July 2010.

[41] R. Ganesh, and J. J. Kumari, "A survey on channel estimation techniques in mimo-ofdm mobile communication systems," International Journal of Scientific Engineering Research vol. 4, no. 5, pp. 1850-1855, 2013.

[42] D. C. Araujo, T. Maksymyuk, A. L. de Almeida, T. Maciel, J.C. Mota, and M. Jo, "Massive MIMO: survey and future research topics," Iet Communications, vol. 10, no. 15, pp. 1938-1946, 2016. 